\title{
Literatura científica sobre análisis estadístico implicativo: un mapeo sistemático de la década que transcurre.
}

\section{Scientific literature on Implicative Statistical analysis: a systematic mapping of the decade that passes.}

Bryan Marcelo Barragán Pazmiño. ${ }^{1}$ \& Rubén Antonio Pazmiño Maji. ${ }^{2}$.

Recibido: 08-07-2017 / Revisado:17-7-09-2018 Aceptado: 05-10-2018/ Publicado: 01-11-2018

\begin{abstract}
.
DOI: https://doi.org/10.33262/cienciadigital.v2i4.1..190

Statistical Implicative Analysis every day makes its way as one of the most important analyzes when it comes to finding statistical implications or quasi-implications. Although it is not a new technique, it has recently become relevant, since it is an adequate methodology to work in big data. The present study was carried out in order to know the situation of Statistical Implicative Analysis according to the amount of scientific production produced so far this decade, through the analysis of 121 documents obtained through a systematic procedure of inclusion and exclusion criteria of documents. It was concluded that Statistical Implicative Analysis is mostly applied in Education's field, with higher production by researchers from France, and with more than half of publications in French, however, it was observed that documents in other languages than French are growing in number, as in other fields of application apart from education and with a greater number of countries in which new scientific production is being produced using Implicative Statistics.
\end{abstract}

Keywords: Systematic Mapping Review, Scientific Literature, Statistical Implicative Analysis.

\footnotetext{
${ }^{1}$ Escuela Superior Politécnica de Chimborazo, Grupo de Investigación en Ciencia de Datos, Riobamba, Ecuador, bryan.barragan@espoch.edu.ec

${ }^{2}$ Escuela Superior Politécnica de Chimborazo, Grupo de Investigación en Ciencia de Datos, Riobamba, Ecuador,rpazmino@espoch.edu.ec
} 


\section{Resumen.}

El Análisis Estadístico Implicativo cada día se abre camino como uno de los análisis más importantes cuando se trata de hallar implicaciones estadísticas o cuasiimplicaciones. Si bien no es una técnica nueva, esta ha cobrado relevancia recientemente, pues es una metodología adecuada para trabajar en grandes cantidades de datos. El presente estudio se realizó con el fin de conocer la situación del Análisis Estadístico Implicativo según la cantidad de producción científica producida en lo que va de la década, mediante el análisis de 121 documentos obtenidos mediante un procedimiento sistemático de criterios de inclusión y exclusión de documentos. Se concluyó que el Análisis Estadístico Implicativo es mayormente aplicado en el campo de la educación, con mayor producción realizada por investigadores de Francia, y con más de la mitad de publicaciones en francés, sin embargo, se observó que los documentos en idiomas distintos al francés van creciendo en número, al igual que documentos en otros campos de aplicación a parte de la educación y con un mayor número de países en los que se está realizando nueva producción científica usando la Estadística Implicativa.

Palabras Claves: Revisión De Mapeo Sistemático, Literatura Científica, Análisis Estadístico Implicativo

\section{Introducción .}

El Análisis Estadístico Implicativo (ASI, por sus siglas en francés) ha venido siendo desarrollado principalmente por Regis Gras, Jean-Claude Régnier y colaboradores desde finales de los 70, con el objetivo de definir un campo teórico que se encuentre enfocado en el concepto de la cuasi-implicación o implicación estadística, para así diferenciarlo de conceptos como las implicaciones lógicas y matemáticas (Gras, Regnier \& Guillet, 2009a; Ferreira, 2015).

El ASI surge a partir de situaciones referentes a la enseñanza de matemáticas, estructurando información, interrelacionando individuos y variables en estudio, generando reglas de asociación entre las variables y en base a estas, la explicación según una determinada situación (Gras, 1979; Gras \& Kuntz, 2009). Los cocientes estadísticos que entran en juego al usar ASI pueden ser: intensidades de implicación, cohesión de clases, implicacióninclusión, significación de niveles jerárquicos, contribución de variables suplementarias, uso de variables modales, binarias, de intervalo y difusas, etc (Gras, Regnier, Lahanier, et al, 2017). En general, una pregunta para ser resuelta por el ASI se puede indicar como: "¿en qué medida se puede considerar que de cumplirse la variable a implica que se cumpla la variable b, sin que se deba al azar?” (Gras \& Regnier, 2009). 
El procedimiento propuesto por Gras en 1992 es resumido por Lacasta en las actas del I Simposio de la Sociedad Española de Investigación en Educación Matemática de la siguiente manera (Rico \& Sierra, 1998):

- Elección del método de análisis

- Elaboración de una tabla de datos adecuada para la aplicación del método elegido

- Definición del conocimiento matemático adecuado para la realización del análisis e interpretación de los resultados

- Interpretación de resultados numéricos y gráficos, a manera de resumen.

- Confirmación o validación mediante un análisis confirmatorio

- Aplicación de un método inferencial

Durante los últimos 30 años se ha venido desarrollando la teoría del ASI estimulada por la interacción entre esta y las aplicaciones que se le ha dado, presentando todavía su mayor campo de acción en la educación, pero evolucionando a otros campos como la psicología, sociología, informática, medicina, etc., demostrando así la capacidad y el potencial del ASI en comparación con otros métodos usados con el mismo objetivo, pero también estableciendo límites en su aplicabilidad debido al concepto de cuasi-implicación (Gras, Regnier \& Guillet, 2009b; De Andrade, Regnier \& Andrade, 2017).

La revisión sistemática es una metodología que tiene como objetivo principal dar un perfil de lo que sucede con cierta área de investigación, mediante el análisis de la producción científica en la misma, pero con el plus de utilizar la información adecuada para dicho análisis (criterios de calidad, inclusión y exclusión de archivos). Esta forja sus cimientos en estudios sobre medicina, pero con una actual ampliación en sus campos de aplicación, siendo uno de ellos el de la Estadística (González, Urrútia \&Alonso, 2011; Liberati, Altman, Tetzlaff, et al, 2009).

Las características que toda revisión sistemática debe poseer son (Vidal, Oramas \& Borroto, 2015):

- Rigurosas: en cuanto a criterios usados para definir la inclusión, exclusión y/o calidad del documento.

- Informativas: deben tratar un problema claramente definido en tiempo y espacio, con el fin de que los resultados permitan una toma de decisiones adecuada.

- Exhaustivas: deben usar la mayor cantidad de información pertinente al tema de análisis.

- Explícitas: deben explicitarse teórica y prácticamente los métodos usados en la revisión. 
Las revisiones sistemáticas se pueden clasificar, según la profundidad del estudio, en dos: las revisiones sistemáticas de literatura, que son estudios más estructurados con relación a los criterios de selección y evaluación de los documentos analizándolos más a fondo; y los mapeos sistemáticos de literatura, los cuales dan una visión general del panorama actual del tema en análisis (Marques, 2017). La metodología para realizar una revisión sistemática se define de manera sintética en la Figura 1., y está basada en Manual de Revisores del Centro Cochrane Iberoamericano (2001).

La revisión narrativa en general, es un proceso de observación, selección y ejecución de un análisis para resumir características de interés en un gran conjunto de documentos, mientras que la revisión sistemática, y en este caso en particular el mapeo de literatura científica, tienen el mismo objetivo, con una diferencia aparentemente simple, pero muy significativa; la sistematización de criterios de selección, inclusión y exclusión de documentos, con el fin de trabajar con información de un área específica, que debe llevarse a cabo bajo un control riguroso y así evitar sesgos de información (Pazmiño, García \& Conde, 2016; García, 2017). Esta diferencia se vuelve clave en la realización del estudio, ya que estos filtros dan mayor peso a los resultados descriptivos de la investigación, sin embargo, en ningún caso se puede generalizar esta clase de resultados sin análisis confirmatorios de posteriores estudios que lo repliquen. Entonces, el presente estudio pretende esclarecer el panorama que ha atravesado el ASI en los últimos siete años (2011 - 2017), según el número de artículos científicos, actas y ponencias de conferencias, capítulos de libros, presentaciones en congresos o coloquios y demás documentos que consideran métodos de implicación estadística según su campo de aplicación, idioma y lugar de elaboración.

\section{Materiales y Métodos.}

Con el fin de determinar la situación de los estudios que tienen al menos una aplicación de ASI dentro de su contenido, desde el año 2011 a 2017, se realiza el mapeo de dicha literatura con la metodología de las revisiones sistemáticas en general, cuyos pasos se detallan en la Figura 1., y que se describen a continuación con su respectiva aplicación a la presente investigación hasta el paso 4, que corresponde a la parte metodológica del mapeo sistemático. Los pasos 5 y 6 de esta metodología referentes al análisis y presentación e interpretación de resultados respectivamente, se discutirán en las secciones de Resultados y Discusión del presente escrito. 


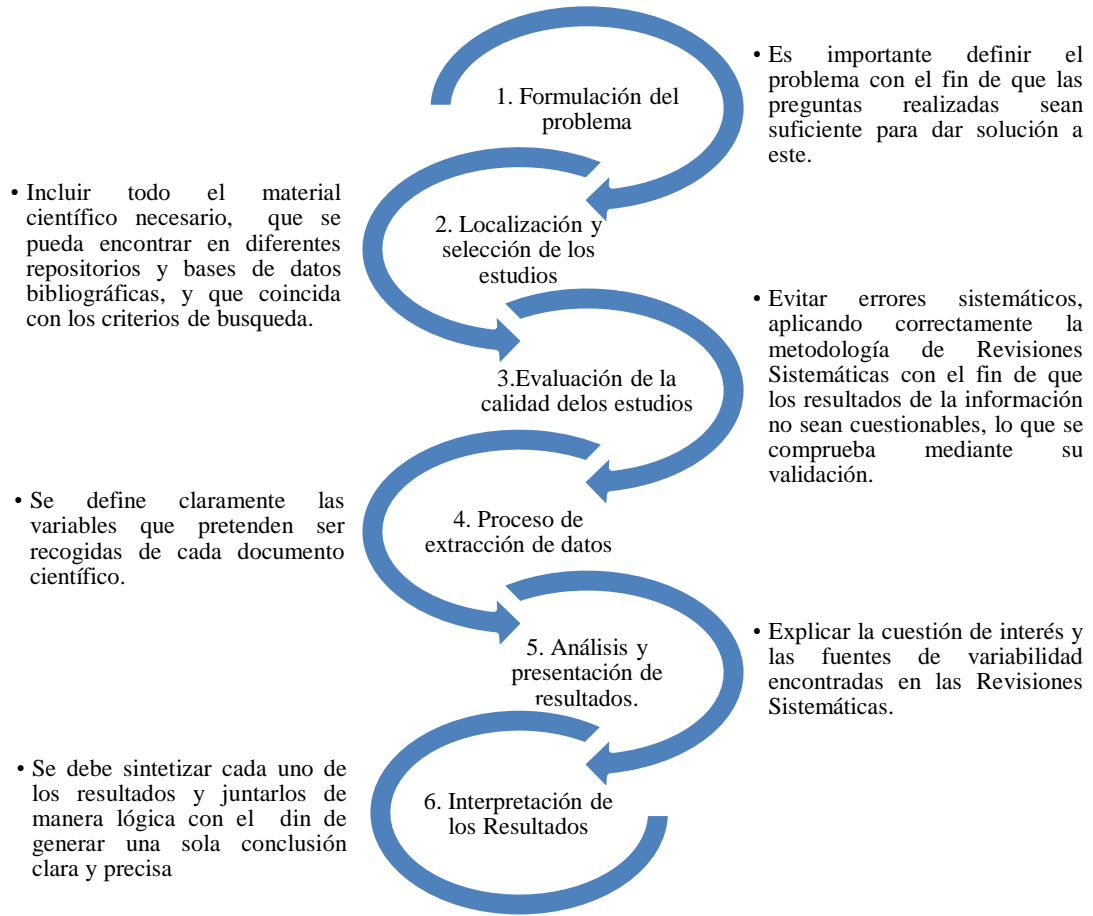

Figura 1. Metodología de las Revisiones Sistemáticas. Fuente: (De Dios \& Santamaría, 2007).

1. Formulación del problema. El estudio tiene por meta conocer el estado actual de las publicaciones científicas de ASI, con información de la década en curso.

2. Localización y selección de los estudios. Se definieron varias bases de datos bibliográficas como fuente para encontrar la información referente a ASI desde el año 2011: IEEE Explorer, Web of Science, Science Direct, Springer, SciELO, La Referencia, Dialnet, EBSCO, ACM, LatIndex, DartEurope, CSIC, PDQT, TDR, DOAJ y ACM; además se toma en cuenta los Coloquios Internacionales de ASI 6, 7, 8 y 9 realizados en los años 2012, 2013, 2015 y 2017 respectivamente. Además, como criterio adicional se consideró solamente a los documentos de libre acceso en la web. Se encontraron 121 documentos con los criterios antes mencionados, los cuales se detallan en la Tabla 1, listando los artículos encontrados en cada repositorio de documentos (los repositorios que no se encuentran listados no tenían documentos sobre ASI). 
Tabla 1.Artículos de ASI publicados desde el año 2011 hasta 2017

\begin{tabular}{|c|c|}
\hline Fuentes & $\mathrm{N}^{\circ}$ de artículos \\
\hline Web of Science & 6 \\
\hline Science Direct & 4 \\
\hline DOAJ & 2 \\
\hline Dart Europe & 3 \\
\hline SciELO & 2 \\
\hline La Referencia & 2 \\
\hline $\mathrm{ACM}$ & 2 \\
\hline A.S.I. 6 & 14 \\
\hline A.S.I. 7 & 18 \\
\hline A.S.I. 8 & 33 \\
\hline A.S.I. 9 & 35 \\
\hline
\end{tabular}

3. Evaluación de la calidad de los estudios. La metodología de la revisión sistemática y de mapeo de literatura es adecuada, excepto en la elección de documentos de libre difusión en la red solamente.

4. Proceso de Extracción de Datos. Para el registro de información en los documentos seleccionados se registró la información de las siguientes preguntas.

a) ¿En qué año fue publicado el documento?

b) ¿El documento se aplica en el ámbito de la educación?

c) ¿El documento se aplica en el ámbito de la psicología?

d) ¿El documento se aplica en el campo de la medicina?

e) ¿El documento se aplica en otro campo?

f) ¿El documento tiene algún aporte teórico?

g) ¿El documento fue redactado en español?

h) ¿El documento fue redactado en inglés?

i) ¿El documento fue redactado en portugués?

j) ¿El documento fue redactado en francés?

k) ¿El documento fue redactado en otro idioma?

1) ¿En qué país se encuentra la institución de afiliación del investigador principal del estudio?

Solamente en la pregunta $l$ ) se observa una variable modal en los datos, mientras que el resto de las variables que se analizan son de carácter binario.

Definidas las preguntas de investigación, resta la recopilación de la información, mediante el análisis de cada uno de los documentos seleccionados según los criterios antes mencionados.

\section{Resultados.}

Como se observa en la Tabla 1., la mayor parte de los artículos referentes a ASI, es presentada en los Coloquios organizados para este tema en específico. Mientras que la información obtenida de repositorios de bases de datos bibliográficas analizados, aún no contienen una 
cantidad importante de documentos de ASI entre su repertorio de documentos científicos.

En cuanto a los resultados de las preguntas relacionadas con el campo de aplicación del ASI, se resumen gráficamente en la Figura 2., indicando que el campo de acción preponderante en el uso de este método sigue siendo la educación con 63 del total de documentos analizados, seguido de los que aportan al desarrollo de la teoría del ASI con 29 documentos, y este seguido por otros campos de aplicación con 15 documentos, mientras que los estudios relacionados con la salud física y mental se ubican al final de esta lista con siete documentos cada uno.

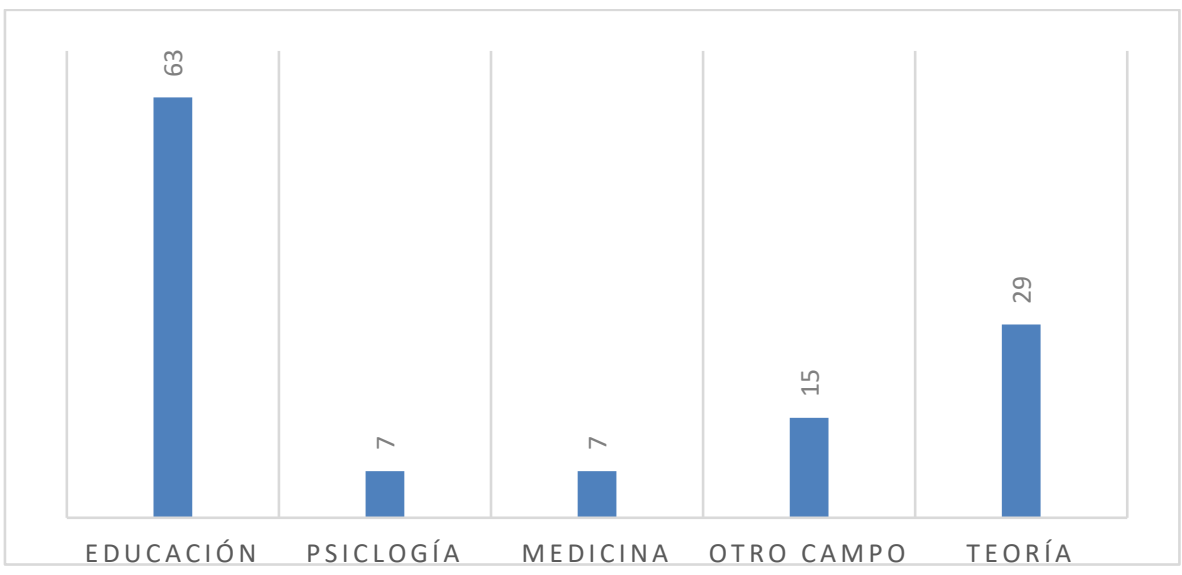

Figura 2. Artículos de ASI según su campo de Aplicación.

Acerca del idioma principal en el que se redactaron los documentos que ocupan ASI (Figura 3.), el francés es en el que se realiza la mayor parte de los documentos de ASI con 66 documentos, seguidos por los documentos en el idioma portugués con 26 y ya algo lejos la producción de ASI en inglés y español con 17 y 12 documentos respectivamente. Es relevante mencionar que no existe producción científica en otros idiomas a los antes mencionados.

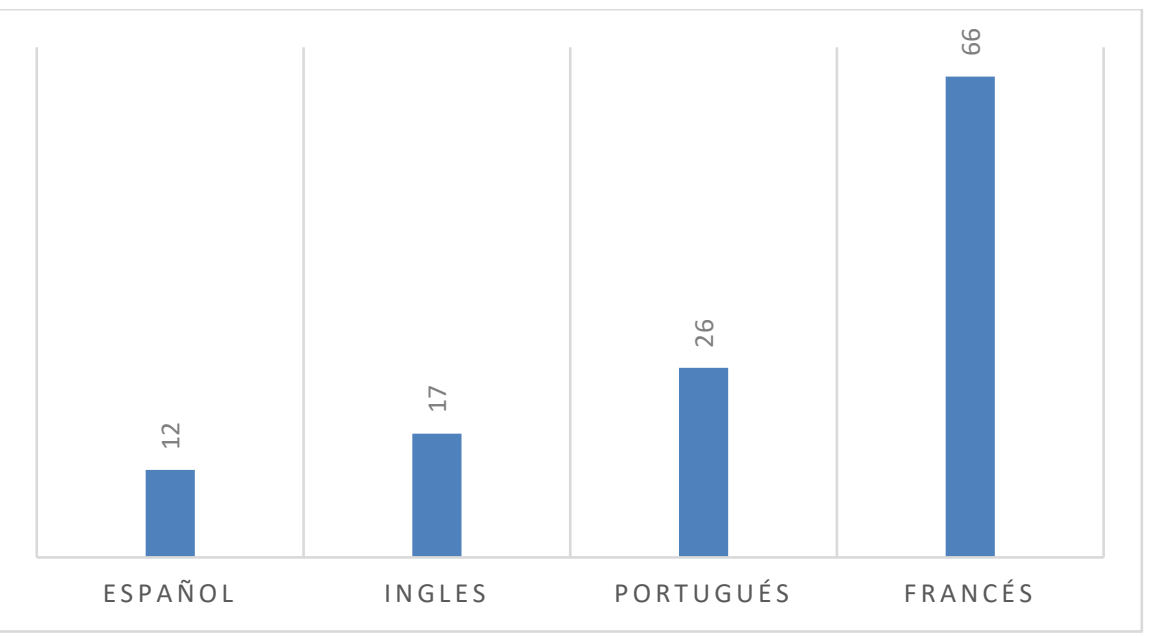

Figura 3. Artículos de ASI según el idioma del documento 
Como se observa en la Figura 4., la producción de ASI en su mayoría es desarrollada o aplicada por investigadores afiliados a instituciones en Francia con 62 documentos, seguido de los documentos producidos en Brasil con 22 documentos y después España con nueve documentos. Argelia, Chipre y Ecuador les siguen a estos con cuatro documentos cada uno, luego se observan a los países de Cuba, Italia, México, Nueva Zelanda y Vietnam con dos documentos cada uno y finalmente los países que han producido un artículo ASI durante el periodo 2011 - 2017, Argentina, Colombia, Gabón, Grecia, Madagascar y Túnez.

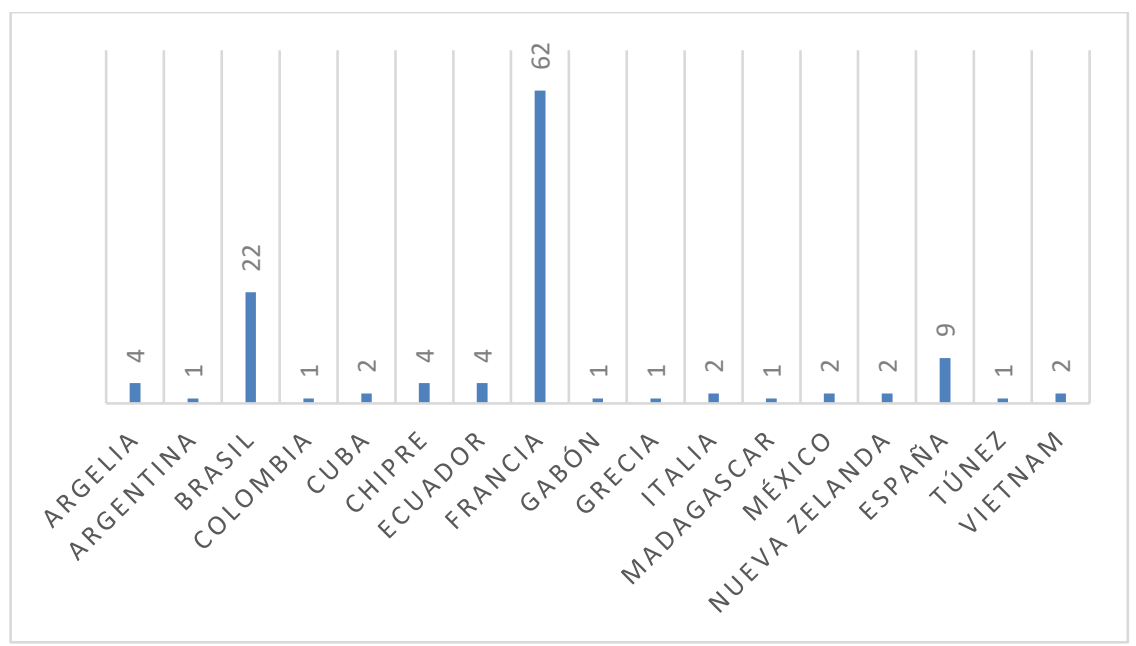

Figura 4. Artículos de ASI según el país de afiliación del autor.

En la Figura 5., se observa la evolución de los documentos del ASI a en lo que va de la década, siendo la del año 2011 muy baja con tres documentos, mientras que en el año 2012 y 2013 aumentó significativamente con 22 y 21 documentos respectivamente, para el año 2014 nuevamente se redujo la producción a un solo artículo, mientras que en el 2015 presentó un aumento notable con 35 documentos, en 2016 disminuyó a cuatro documentos y en 2017 nuevamente se incrementó a 35 .

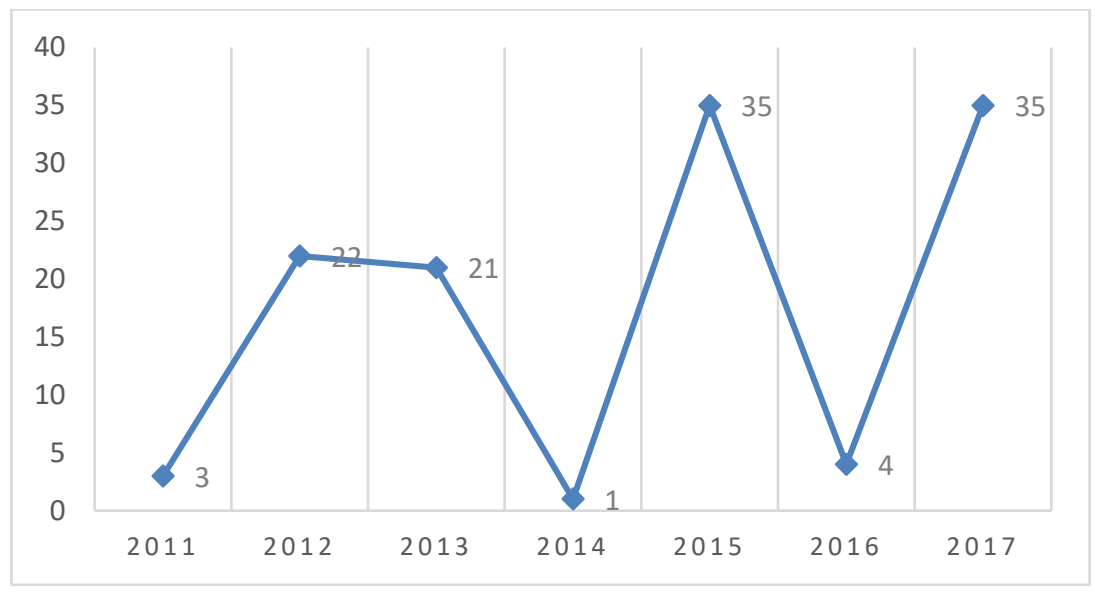

Figura 5. Artículos de ASI durante los últimos 7 años. 
En cuanto a los documentos que presentan un mayor aporte, después de realizada esta revisión de literatura, están: "Genesis and development of statistical implicative analysis: historical retrospective" de Regis Gras (2013), documento redactado en francés, con contenido explicativo de gran aporte teórico para el desarrollo del ASI, sustentado con ejemplos prácticos en los que se muestra explícitamente gráficas e índices usados en esta metodología. Otro documento teórico de gran valía para el ASI es "Dualité Entre Espace Des Variables Et Espace Des Sujets En Analyse Statistique Implicative" de Regis Gras, con colaboración de Dominique Lahanier (2012), escrito en francés, cuyo contenido se asemeja al documento antes mencionado, pero haciendo mayor énfasis en el tratamiento de variables binarias. "A Paradigmatic Survey Of The Implicative Statistical Analysis" de Regis Gras nuevamente (2015), quien esta vez hace énfasis en la aplicación del ASI en el campo educativo con técnicas y gráficos detallados de una manera clara y concisa, documento escrito en francés. Por último, otros dos documentos que explican de manera adecuada la aplicación de índices, técnicas y gráficas del ASI son "Professional master's degree in teaching of mathematics: articulation of competences" de Isaisa de Aguiar y colaboradores (2015), y "Pesquisas Sobre Professores Que Ensinam Matemática Nos Anos Iniciais Do Ensino Fundamental No Brasil Estudos Das Tendências De Pesquisa No Brasil (2006 2014)1 À Luz Da Análise Estatística Implicativa" de Luciana Amaral y colaboradores (2015), son documentos escritos en portugués, los cuales presentan un enfoque del ASI netamente en el plano educativo con el análisis de variables binarias y aplicación de diferentes procedimientos del ASI para encontrar índices y reglas de implicación y asociación.

\section{Discusión.}

El ASI mantiene su fuerte en los estudios y países que le dieron origen, pues la mayoría de los documentos que aplican este método son documentos enfocados en el campo del desarrollo del aprendizaje, en sujetos de estudio que cursan la educación básica y, particularmente, entre la relación de las técnicas de enseñanza implementadas con el éxito o fracaso en cuanto al aprendizaje de dichos sujetos (Lacasta, Lasa \& Wilhelmi, 2012; Almouloud, Coutinho \& Da Silva, 2015; De Menezes \& Dos Santos, 2015); sin embargo, se observa también una ampliación en los campos de acción del ASI, pues se definen otros campos como el de la psicología y medicina en los que se ha venido aplicando últimamente, para encontrar relaciones entre que tan adecuado resultan ciertos tratamientos de diferentes trastornos físicos y psíquicos que poseen los pacientes estudiados (Pasquier \& Gras, 2012; Ndong, 2012).

De igual manera sucede con el idioma de escritura original de los documentos, pues la mayoría se encuentran redactados en el idioma francés, no obstante, se cuenta con una cantidad considerable de documentos en portugués, y se observa un valor ciertamente notable en los documentos en español e inglés, lo que en cierto modo indica la diseminación que está 
teniendo el ASI en varias partes del mundo (Pellois, 2017; Da Costa A., Da Costa C., Vieira, et al, 2017; Huerta, Couturier \& Pazmiño, 2013; Pérez, Pazmiño \& Andaluz, 2014). Esta proposición se corrobora observando los países de las instituciones de afiliación de los investigadores que se están introduciendo en la aplicación de técnicas de ASI, observando seis países con un documento y cinco países con dos documentos cada uno, mientras que este parámetro es liderado por Francia, Brasil y algo lejos por España (Nguyen, Huynh H., Huynh X, et al, 2017; Lurato, 2012; Carranza, 2013; Totohasina, 2014).

La evolución de la cantidad de documentos de ASI en el tiempo es algo curiosa de observar, pues se definen crecimientos y decrementos muy abruptos de esta característica dependiendo del año, comportamiento que se debe a que la mayoría de estos documentos son presentados en los Coloquios de ASI, los cuales en esta década se han realizado en los años 2012, 2013, 2015 y 2017, que coinciden con los años de mayor cantidad de documentos de ASI encontrados. En dichos coloquios se observa que el desarrollo teórico de esta rama se sigue realizando, aunque cada vez con menor énfasis en esta y más en su aplicabilidad, como lo muestran las actas de dichos coloquios, que en el 6to Coloquio basa su temática mayormente en el desarrollo teórico-practico del ASI, mientras que en el 7mo ya se enfatiza en la confirmación de la teoría desarrollada, luego en el 8vo y 9no se observa que el tópico principal es la ampliación aplicativa del ASI, ya no solamente en ciencias básicas, sino también en las sociales. Además, se observa la expansión del ASI puesto que las convenciones han tenido su lugar de realización ya no solamente en Europa, como se venía acostumbrando en los coloquios realizados el decenio anterior, sino que se trasladaron hacia Sudamérica (Brasil) y África (Túnez) donde se desarrollaron el 6to y 8vo Coloquio respectivamente (Regnier, Gras \& Bailleul, 2012; Regnier, Almouloud \& Gras, 2013; Regnier, Slimani \& Gras, 2015; Regnier, Gras, Couturier, et al, 2017).

\section{Conclusiones.}

El esparcimiento de las técnicas y métodos que ofrece el ASI para determinar implicaciones estadísticas o cuasi-implicaciones se ve segregado de los estudios que ocasionaron su surgimiento, pues si bien se observa que la mayor cantidad de documentos de ASI se encuentran elaborados en Francia y en idioma francés, la cantidad de investigadores de diferentes países que han aplicado esta técnica es considerable para un método que está cobrando una importancia desde hace muy poco tiempo relativamente. Este comportamiento resulta excelente para la dispersión del conocimiento del ASI, pues diferentes organizaciones a nivel mundial están familiarizándose con los beneficios que las técnicas que esta metodología ofrece en comparación con otras que están enfocadas en la misma dirección, pero que no tienen la misma eficiencia (Orús, Zamora \& Huerta, 2009). Se espera que en lo que resta de década las publicaciones de ASI sigan presentando el mismo comportamiento de incremento cuando se realizan Coloquios y decremento cuando no, pero para un futuro algo más lejano, que se comience a producir un crecimiento en la utilización de las técnicas 
de ASI. Se determinó que, en su mayoría, los documentos que aplican ASI están escritos en francés, por investigadores afiliados a instituciones francesas y que aún encuentra en la educación su mayor campo de aplicación, pero con número considerable en otros campos de aplicación, en otros idiomas y en instituciones de otros países.

\section{Agradecimiento.}

Nos gustaría brindar un elocuente agradecimiento al Grupo de Investigación en Ciencia de Datos - CIDED, de la Escuela Superior Politécnica de Chimborazo, el cual nos ha permitido aprender e implementar nuevas técnicas y métodos para la búsqueda, el preprocesamiento y el análisis de información, además de la confianza y el ambiente cordial brindado en su seno, por parte de todos los investigadores y directivos que lo integran.

\section{Referencias bibliográficas.}

Almouloud, S., Coutinho, C. \& Da Silva, M. (2015). Mapeamento de conhecimentos de professores sobre tecnologias de informação e comunicação e seus usos didáticopedagógicos. Ou leur parrainage scientifique, 560.

Amaral, L., Câmara Dos Santos, M., Acioly, N. \& Edênia M. (2015). Pesquisas Sobre Professores Que Ensinam Matemática Nos Anos Iniciais Do Ensino Fundamental No Brasil Estudos Das Tendências De Pesquisa No Brasil (2006 - 2014)1 À Luz Da Análise Estatística Implicativa.

Carranza, P. (2013). Presencia de interpretaciones bayesiana y frecuentista de la probabilidad en libros de estudio en Francia.

Centro Cochrane Iberoamericano. (2001). Manual de revisores (versión española de "Cochrane Reviewers Handbook"). Barcelona.

Da Costa, A., Da Costa, C., Vieira, M., Eloi, Q., de Andrade, V. \& Regnier, J. C. (2017). Estudo exploratório do pensamento geométrico de professores de matemática: caso de um grupo na PARAÍBA (BRASIL) a luz da ASI.

De Aguiar, S., Bisognin, E., Bisognin, V., Régnier, J. C., Acioly, N. \& Cardoso, A. (2015). Professional master's degree in teaching of mathematics: articulation of competences.

De Andrade, V., Regnier, J. C. \& Andrade, P. (2017). As produções na área de Ensino de ciências e matemática nas comunicações nos colóquios internacionais sobre Análise Estatística Implicativa.

De Dios, J., Santamaría, A. (2007). Revisión sistemática y metanálisis (I): Conceptos 
básicos. Evidencias en pediatría; 3(4); 21.

De Menezes, M. \& Dos Santos, M. (2015). O saber escolar na perspectiva da Teoria Antropológica do Didático.

Ferreira, L. (2015). A formação humana dos educadores através das comunicações nos colóquios internacionais sobre análise estatística implicativa (ASI): casos dos colóquios ASI 5, ASI 6 e ASI 7. Ou leur parrainage scientifique, 602.

García, F. (2017). Mapeos sistemáticos de literatura, revisiones sistemáticas de literatura y benchmarking de programas formativos.

González, I., Urrútia, G. \& Alonso-Coello, P. (2011). Revisiones sistemáticas y metaanálisis: bases conceptuales e interpretación. Revista española de cardiología; 64(8); 688-696.

Gras, R. (1979) Contribution à l'étude expérimentale et à l'analyse de certaines acquisitions cognitives et de certains objectifs didactiques en mathématiques [tesis doctoral]. Université de Rennes.

Gras, R., Régnier, J. C. \& Guillet, F. (2009). Analyse Statistique Implicative. Une méthode d'analyse de données pour la recherche de causalités.

Gras, R.\& Regnier, J. C. (2009). Fondements théoriques de l'analyse statistique implicative.

Gras, R. \& Kuntz, P. (2009). El Análisis Estadístico Implicativo (ASI) en respuesta a problemas que le dieron origen. Teoría y aplicaciones del Análisis Estadístico Implicativo: primera aproximación en lengua hispana. Castellón: Departamento de Matemática de la Universitat Jaume I: 3-51.

Gras, R. \& Lahanier, D. (2012). Dualité Entre Espace Des Variables Et Espace Des Sujets En Analyse Statistique Implicative.

Gras, R. (2013). Genesis and development of statistical implicative analysis: historical retrospective.

Gras, R. (2015). A Paradigmatic Survey Of The Implicative Statistical Analysis.

Gras, R., Regnier, J. C., Lahanier-Reuter, D., Marinica, C. \& Guillet, F. (2017). L'analyse statistique implicative. Des Sciences dures aux Sciences Humaines et Sociales. Cépaduès Editions: 462.

Huerta, P., Couturier, R. \& Maj1, R. (2013). On the probability distribution of the classical gras implication index between two binary random variables. In VII International 
Conference on Statistical Implicative Analysis, Brazil.

Lacasta, E., Lasa, A. \& Wilhelmi, M. R. (2012). Actividad lógica y relacional en Educación Infantil.

Liberati, A., Altman, D., Tetzlaff, J., Mulrow, C., Gøtzsche, P., Ioannidis, J., et al. (2009). The PRISMA statement for reporting systematic reviews and meta-analyses of studies that evaluate health care interventions: explanation and elaboration. PLoS medicine; $6(7)$.

Lurato, G. (2012). The implicative statistical analysis: an interdisciplinary paradigm.

Marques, M. (2017). Systematic Review. Investigación en Ciencia de la Computación.

Ndong, L. (2012). Malnutrition protéino-énergétique et enseignement de la nutrition et de l'alimentation au Gabon. L'Analyse Statistique Implicative: de l'exploratoire au confirmatoire, 263-289.

Nguyen, H., Huynh, H. H., Huynh, H. X. \& Couturier, R. (2017). Recommended based on asymmetric user relations using timp (temporal implicative) measure.

Orús, P., Zamora, L. y Huerta, P. (2009). Teoría y aplicaciones del Análisis Estadístico Implicativo: primera aproximación en lengua hispana.

Pasquier, D. \& Gras, R. (2012). De l'intérêt de l'analyse statistique implicative (ASI) pour la recherche exploratoire en psychologie. Psychologie française, 57(3), 161-173.

Pazmiño, R., García, F. \& Conde-González, M. (2016). Approximation of statistical implicative analysis to learning analytics: a systematic review. In Proceedings of the Fourth International Conference on Technological Ecosystems for Enhancing Multiculturality - ACM; 355-376.

Pellois, C. (2017). Traitement Par L'asi De Modèles En Sciences Humaines : Entre Simples Fluctuations, Artefacts, Et Sens Possibles.

Pérez, M., Pazmiño, R. \& Andaluz, V. (2014). Cuasi-implicación estadística y determinación automática de clases de equivalencia en imágenes de resonancia magnética de cerebro. Revista Politécnica, 34(1), 123.

Regnier, J. C., Gras, R. \& Bailleul, M. (2012). De l'exploratoire au confirmatoire. 6ème Colloque d'Analyse Statistique Implicative: 7, 8, 9 y 10 novembre 2012, Sao Paulo, Brasil.

Regnier, J. C., Almouloud, S. \& Gras, R. (2013). Cadre théorique et applicatif pour 
l'exploration sématique et non symétrique des donn'ees. 7ème Colloque d'Analyse Statistique Implicative: 27, 28, 29 y 30 novembre 2013, Tunisia.

Regnier, J. C., Slimani, Y. \& Gras, R. (2015). Des sciences dures aux sciences humaunes et sociales. 8ème Colloque d'Analyse Statistique Implicative: 11, 12, 13 y 14 novembre 2015.

Regnier, J. C., Gras, R., Coururier, R. \& Bodin, A. (2017). Points de vue conceptuels, applicatifs et métaphoriques. En: 9ème colloque d'Analyse Statistique Implicative: 4, 5, 6 y 7 octobre 2017, Belfort, France.

Rico, L. \& Sierra, M. (1998). Primer simposio de la Sociedad Española de Investigación en Educación Matemática; 1997 Sep 12, 13; Salamanca; Universidad de Salamanca.

Totohasina, A. (2014). Towards a theory unifying implicative interestingness measures and critical values consideration in MGK. Educação Matemática Pesquisa: Revista do Programa de Estudos Pós-Graduados em Educação Matemática, 16(3), 881-900.

Vidal, M., Oramas, J. \& Borroto, R. (2015). Revisiones sistemáticas. Educación Médica Superior; 29(1); 198-207.

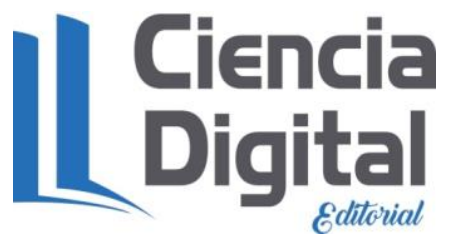




\section{Para citar el artículo indexado.}

Barragán, B. \& Pazmiño, R. (2018). Literatura Científica sobre Análisis Estadístico Implicativo: Un mapeo sistemático de la década que transcurre. Revista electrónica Ciencia Digital 2(4.1.), 55-69. Recuperado desde: http://cienciadigital.org/revistacienciadigital2/index.php/CienciaDigital/article/view/190/16 $\underline{8}$

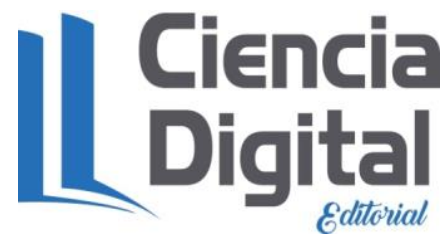

El artículo que se publica es de exclusiva responsabilidad de los autores y no necesariamente reflejan el pensamiento de la Revista Ciencia Digital.

El articulo queda en propiedad de la revista y, por tanto, su publicación parcial y/o total en otro medio tiene que ser autorizado por el director de la Revista Ciencia Digital.
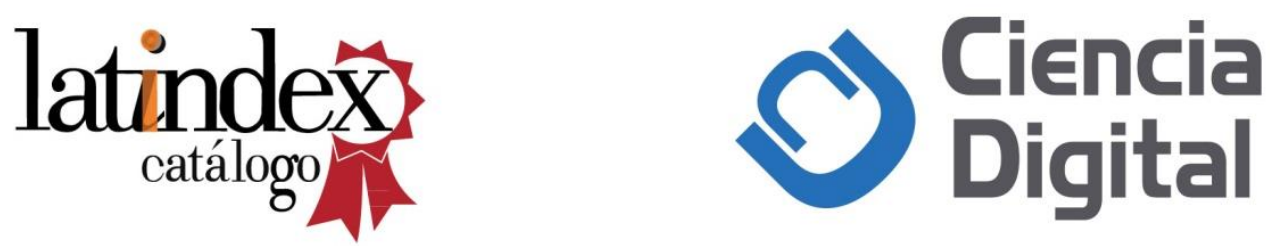